\title{
Improvised Learning for pre-primary students using Augmented Reality
}

\author{
Madhusmita Kalita \\ Std. Id : 1115467 \\ Masters in Comp. Sci. \\ Lakehead University, Thunder Bay \\ Sagar Deshpande \\ Std. Id : 1109819 \\ Masters in Comp. Sci. \\ Lakehead University, Thunder Bay
}

\author{
Simranjeet Singh \\ Std. Id :1093604 \\ Masters in Comp. Sci. \\ Lakehead University, Thunder Bay \\ Tanmay Kank \\ Std. Id : 1110177 \\ Masters in Comp. Sci. \\ Lakehead University, Thunder Bay
}

\author{
Mina Armanyous \\ Std. Id :1117659 \\ Masters in Comp. Sci. \\ Lakehead University, Thunder Bay \\ Dr. Sabah Mohammed \\ COMP5112WC Supervisor \\ Lakehead University, Thunder Bay
}

\begin{abstract}
In this age of digital advancements, where technologies are changing in a fraction of time. From the abacus which made tutoring math easy millennia back, to word processor which changed the way research paper is being written and presented. After every era, with the advancements in the technology has not only given shaped the education but also transformed it. There was a time when the education world of black on white changed to projected presentations. However, now in this paper, we believe to go beyond the two-dimensional space and create a whole new educational world for children. Augmented Reality (AR) has successfully made classroom learning more interactive and engaging for students as well as for teachers to deliver their lectures. $A R$ is the combination of the real-world with computer- generated world. It is one of the most emerging fields in computer science. The conventional approach for learning can be stressful and to a certain extent less effective for some students. So, we propose a system in which students use smart devices like tablets, mobile, etc. that act as an alternative to boring supportive textbooks. Also, we plan to develop an application consisting of two modules like interactive learning and fun examination, a hybrid of the traditional approach and innovative practical illustrations of complicated concepts leading education in another dimension.

Index Terms-Augmented Reality, Technology, Education
\end{abstract}

\section{INTRODUCTION}

Augmented Reality can superimpose real-time computer animation onto the real world created by combining the perceived physical elements in the world around us with computer-based graphics, video, audio, GPS data, etc. It is enriched by adding virtual data in the real world we sense but unlike virtual reality, augmented reality does not often require a separate room or confined area to create an immersive environment. The idea which makes this developing technology a stronger tool to provide toddlers with education is the way it is delivered. What someone may call an 'unsurprising' almost every nine out of ten own smartphones, so why aren't teachers utilizing these in the classroom or campus? To elaborate further, Augmented reality makes use of smart technologies and smart devices to covey knowledge. According to the cone of experience theory, learners only remember $10 \%$ of what they read but remember $90 \%$ of what they say as they perform an action by seeing and doing a simulation experience (Chih, 2007). So, it is rightly proven that audio-visual catalyzes the process of learning. Imagine Augmented reality in various fields like science, can be used in a range of topics in science, allowing students to be provided with guided tours to places like outer space to the depth of the ocean life. Moreover, if implemented in the field of mathematics, it can play a major role in subjects like geometry and calculus, creating its own world of numbers. Also, Augmented reality in gaming uses the existing environment and creates a playing field within it. AR games are typically played on devices like smartphones, tablets and portable gaming systems. An approach of merging these two concepts of gaming and education to interact with the real world in digital form while experiencing the real-life environment giving them a euphoric experience and greater innovations was derived by us.

A game can be implemented in many important fields such as medical training, retail, repair maintenance, military, education, etc. and implementing it in education provides new possibilities. With the benefits of Augmented reality as well as gaming, education can be more varied, unique, personalized and interactive. It helps in turning complex and boring content to be easy and fun and also makes it creative. So, we attempted to implement our research by developing an augmented reality- based education application using Unity 3D Platform and Vuforia SDK, which targets an audience of preschool students (4-9 years). There are many platforms for making AR application and SDK's to choose from, but for our research Unity and Vuforia made a dynamic combination. Likewise, Unity is a 2D and 3D game creation environment that supports social media integration, fantastic graphical support, multiplatform support, and Unity has its own Asset Store. Vuforia is the most widely used platform for AR development, with support for leading phones, tablets, and eyewear. The motive of our application is to use augmented reality to make learning more interactive and fun as well as to keep the application scalable and executed over a wide range of devices. We have developed two modules- Learning, and examination. In the Learning module, children can learn interactively. When users display the flashcard of one of the different alphabet or numbers pictures defined in the application, they see the respective moving 3D augmented object of fruit. This can help the students to remember better and makes learning fun. In testing, Examination will not stress. For each question, users will have to display the answer flashcard which they think is correct. If the student chooses the correct flashcard it will display on- screen as correct. This is expected to help the young minds to capture the alphabet or numbers into their memory easily. 


\section{LITRETURE REVIEW}

Although AR is one of the technologies which can change the scenario in education these days, still the significance of $\mathrm{AR}$ in learning environments remains unclear and unaware [2]. Furthermore, various types of AR applications exist in educational environments, which may differ regarding their benefits towards educational outcomes [1]. From the context of this paper, we refer to educational environments as any outline, in which people are acquiring knowledge in a structured and controlled process.

These are the Five Directions by Yuen et al. [1] which classifies the AR applications into five groups which are referred before carrying out our research in AR. Firstly, AR can be used in applications that enable Discovery-based Learning. A user is provided with information about a realworld place while simultaneously considering the object of interest. Secondly, AR can also be used in Objects Modeling applications. Such applications allow students to receive immediate visual feedback on how a given item would look in a different setting. Thirdly, AR Books are books that offer students 3D presentations and interactive learning experiences through AR technology. The books are augmented with the help of technological devices such as special glasses. Moreover, one idea for the support of training individuals in specific tasks is described by Skills Training. Especially mechanical skills are likely to be supported by AR Skills Training applications. Lastly, video Games offer powerful new opportunities for educators which have been ignored for many years [3]. Nowadays, educators have recognized and often use the power of games in educational environments. AR technology enables the development of games which take place in the real world and are augmented Also, one of the researches that we referred in which the children were taught using the flashcards of different animals on which they see the 3D character defined and even they can hear their voices. This was the best way to bring twodimensional education to the 3-D world which not only provides education in audio-visual format but also creates a whole new virtual world of learning [9]. While, discussing how this works were implemented they used Unity with Vuforia SDK which can be compiled in accordance with different platforms such as Web, IOS, Android, and Windows Phone without any infrastructure changes.

The benefit of Augmented Reality is described in quotations such as "the AR-style gameplay successfully enhanced intrinsic motivation towards the self-learning process" [6], "Participants using the AR books appeared much more eager at the beginning of each session compared with the NAR group" [4], and "students have been satisfied and motivated by these new methodologies, in all cases" [5]. The benefit can be further described by findings such as the users being "more proactive"

[7] [8] or the will to continue learning using the AR technology after class. A more detailed description was found in Iwata et al. [6], where physical interaction is explicitly identified as a driver to enhance emotional engagement.

Since various types of AR applications exist in educational environments, which may differ regarding their benefits towards educational outcomes. For the context of this research, we refer to educational environments as any scenario, in which pre-school children can acquire knowledge in a structured and controlled process. We have seen the idea of developing scientific literacy using augmented reality in preschool education [10]. Several models of data collection are referred here which are collecting opinions, beliefs, explanations, knowledge of subjects involved in Semi-structured interviews and focus group discussions. The following paper consists of the idea of Unstructured Observation, Direct as well as Indirect observations that are used to collect various research data. Selective coding, Continuous comparison, and Open coding methods are used to analyze the collected data. Conceptual labels were assigned to individual events, cases and other occurrences of a given phenomenon. By categories, we mean classes of concepts that were identified when comparison of concepts seemed to indicate they belonged to a similar phenomenon [10]. A lot of research material in the form of video recordings and pictures and photographs was also collected in the research. In indirect observation, video recordings of educational activities were watched several times; significant elements supplying evidence on the development of science literacy of pre-school students using augmented reality were looked for.

One of the researches that we went through [11]that focuses on enhancing the use of AR for the learning experience of kindergarten students while addressing parents' concern that a long-time usage of electronic devices may affect their child's health. They developed an AR mobile application prototype to teach kindergarten students English vocabulary interactively and attractively. It allows kindergarten students to learn English vocabulary in any place and at any time using a mobile device [11]. To address the parents' concern on health, they integrate a monitoring system into the application, which allows the parents to monitor their child's usage and stop the application in real-time online. They include a spelling game using AR is included to enhance students' motivation to learn; they can practice spelling, reading and speaking the words through the AR game [11]. Here, Parents can set a time limit to automatically stop the application, manually stop the application online, and track their kid's learning progress. Such monitoring features can let parents control the application usage of their child and alleviate their concerns on their child's health.

After scrutinizing the researches and analyzing the above important researches we decided to go with education domain for the pre-primary students by building an AR application using Unity and Vuforia.

\section{DESIGN METHODOLOGIES}

Our application is developed for the pre-primary students which makes learning fun and examination no stress. We used the Unity 3D game engine platform and Vuforia SDK for developing this application. Unity 3D game engine platform is developed by Unity Technologies for creating video games. It has a cross-platform development, i.e. games can be made for Android, IOS, PC, PlayStation4 lots of other platforms. Unity also has an asset store that provides lots of inbuilt $3 \mathrm{D}$ and 2D models which can be directly used in the game development process. Besides that, it has tools to add some new features to the engine. Vuforia SDK Is a software development kit that is created by Qualcomm. It uses computer vision algorithms to recognize an object or image 


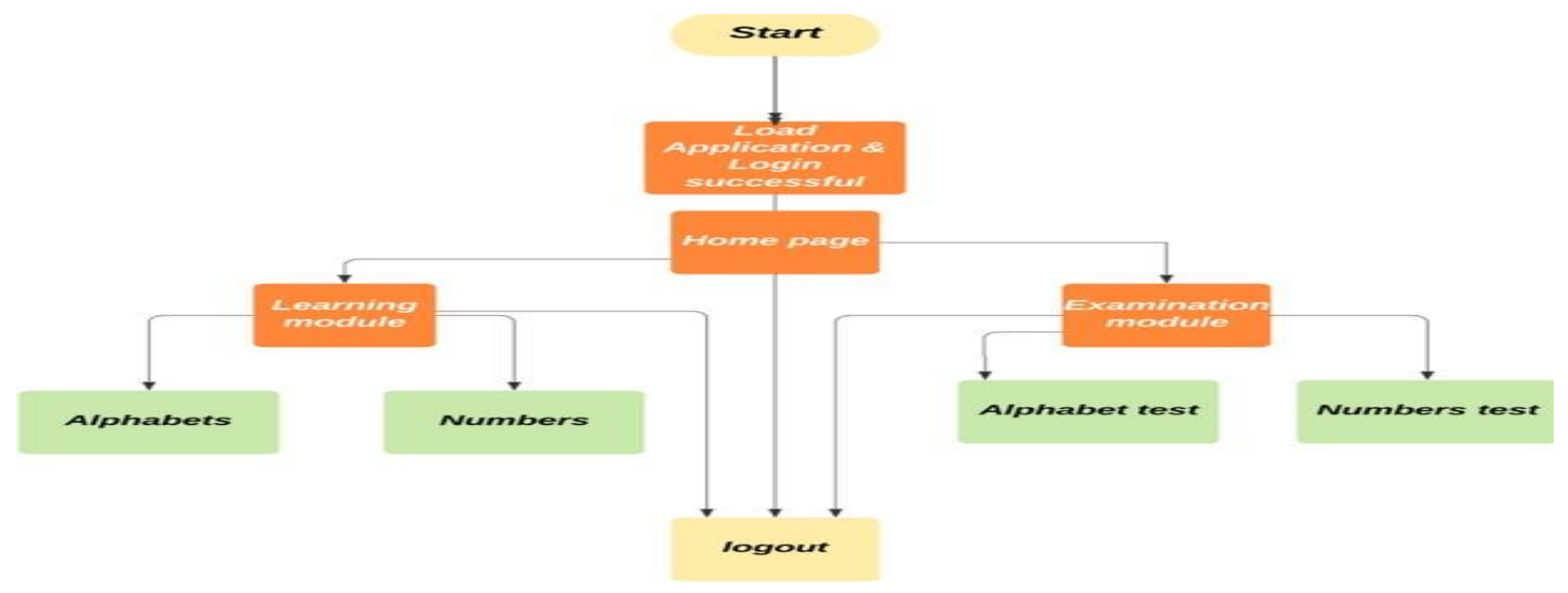

Fig. 1. UI Flow of our application.

or reconfigure the real world. It supports different development environments such as Unity, MS Visual Studio, Apple XCode and Android Studio. Similarly, it supports many devices such as smartphones, tablets and AR smart glasses. Also, it supports building Universal Windows Platform applications for Intel-based Windows 10 devices. It also gives a good user interference. The Fig 1 shows GUI overview of our application. It has two modules "Learning" and" Examination".

\section{A. Learning Module}
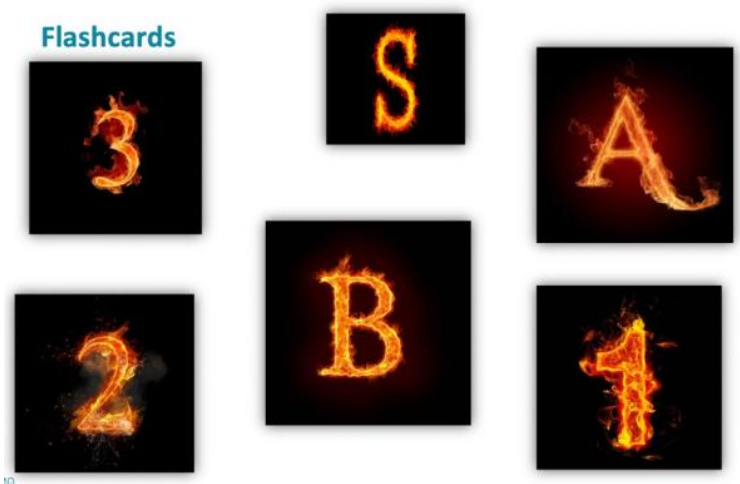

Fig. 2. Flashcards

One of the most important features implemented for this research is the interactive learning module. With the help of this module, children can gain knowledge about new objects. Visualizing these objects in 3D models is the best approach for them to learn. So, the teacher can simply run the application and move the mobile application over the flashcards. These flashcards can be simply for terms that are planned for children to learn such as alphabet or numbers. When the camera gets focused on the flashcard, the application will recognize the image then display the relative virtual 3D model. At the point, children can explore the real object based on the flashcard image. Thus, they can later point to the correct items when they are asked to describe an object based on the alphabets. Flashcard images that have been used for implementation are shown in the Fig 2.

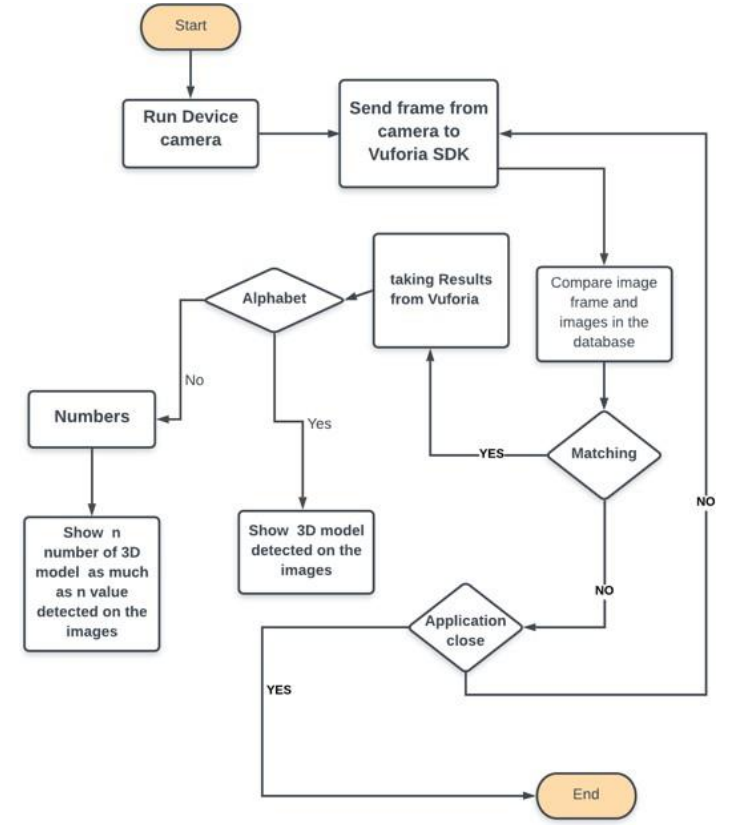

Fig. 3. Flow chart of Learning Module.

Vuforia made the job easier to build this module by providing a set of features that we could apply after integrating Vuforia to Unity 3D studio. So Vuforia lets you define the image targets which will be recognized to render the associated models. Additionally, it allows selecting the 3D model from a list of different packages. In our research, we have used the fruits package to link between alphabet and numbers on one side and fruits on the other side to make the process easier for children to understand. Fig 3 shows the sequence of the Learning module activities.

We wanted our application to be fun and interesting as the audience for this application is children. For this purpose we created rotated and moving the game object.

- Rotation: We used "transform.Rotate" inbuilt function of unity. A game object can rotate around the $\mathrm{X}$ axis, $\mathrm{Y}$ axis, $\mathrm{Z}$ axis. We Used rotation around 20degree Y-axis with a default speed.

- Movement: for moving augmented object we used "trans- form.Translate" function of unity. It moves the transform in the direction and distance of translation. We moved our object forward along its $\mathrm{Z}$ axis i.e. Z:0.5 in 1 unit/second with and default speed. 


\section{Pseudo Code for learning module}

1. START

2. Run device Camera

3. Send image frame from camera to Vuforia SDK

4. If image frame is in Vuforia database

I. Take results from Vuforia

II. If alphabets,

a. Show 3D model detected on the images

b. Rotate the 3D object around 20-degree Y-axis

III. Else, if numbers

a. Detect the number (n)

b. Show n number of 3D model detected.

c. Rotate the 3D object around 20-degree Y-axis

5. Else,

I. If Application close,

II. Else
a. Go to step 6

a. Go to step 3

\section{STOP}

\section{B. Examination Module}

The examination module similar to the learning module is divided into two sub-categories; alphabets and numbers. Since the application is for pre-school children, the question level

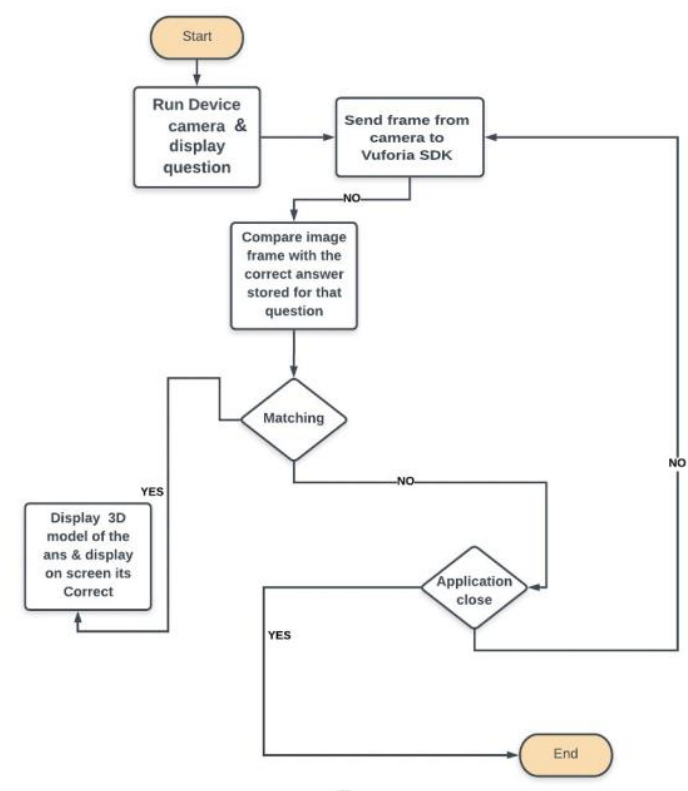

Fig. 4. Flow chart of Examination Module.

will be simple. Alphabet section will handle basic English language questions whereas the number section will have preschool maths. Fig 4. shows the sequence of the examination module activities.
Each target image has a tag attached to it. This tag helps in identifying the target image and the model associated with it. Trackable behavior from Vuforia helps with getting the associated tag, once the tag is received simply compare the received tag with the tag for the correct answer. The image will show a 3D model regardless if the answer is correct or not. However, an event can be raised if the target tag matches the required tag. For our research, we have simply displayed a label raised after the event is triggered. The questions are customizable and can be changed to manage the level of difficulty.

\section{Pseudo Code for examination module}

1. START

2. Display question

3. Run device Camera

4. Send image frame from camera to Vuforia SDK

5. If image frame $=$ correct answer in Vuforia database

I. Take 3D model from Vuforia

II. Show 3D model detected on the images

III. Rotate the 3D object around 20degree Y-axis

IV. Display on screen as "Correct"

6. Else,

I. If Application close,

II.

$$
\text { a. Go to step } 7
$$

a. Go to step 4

7. STOP

\section{Mobile Application Design}

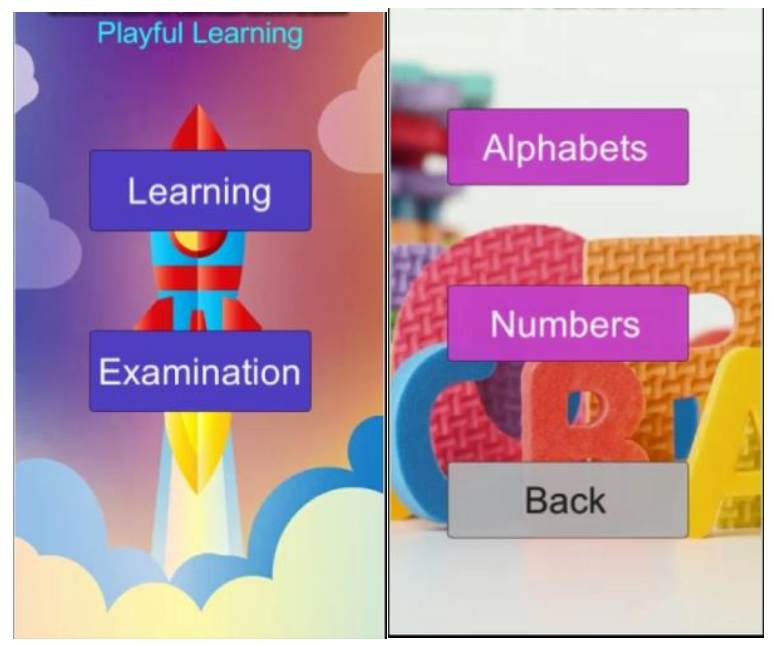

Fig. 5. Sample of application screens

The user interface is simple as shown in fig. 5. The home screen contains two buttons for our two modules; learning and examination. Inside these two modules, there are three buttons for the alphabet, number, and a back button. The back button is to return to the home screen. The alphabet section when 
clicked will activate the AR camera and the alphabet target models whereas the number section will activate the AR camera and the number target models. The difference in learning and examination modules is that the learning module once inside does not have questions whereas the examination module does.

\section{RESULTS}

Our Augmented Reality application had been developed as per the design. Fig 6. Shows an example of learning numbers using the application, so once the camera is focused on the flashcard with the number (3), the application shows three apples. That will simplify understanding the quantitive meaning of the number. Fig. 7,8 show a few basic questions, the trackable behavior from Vuforia helps with the examination.

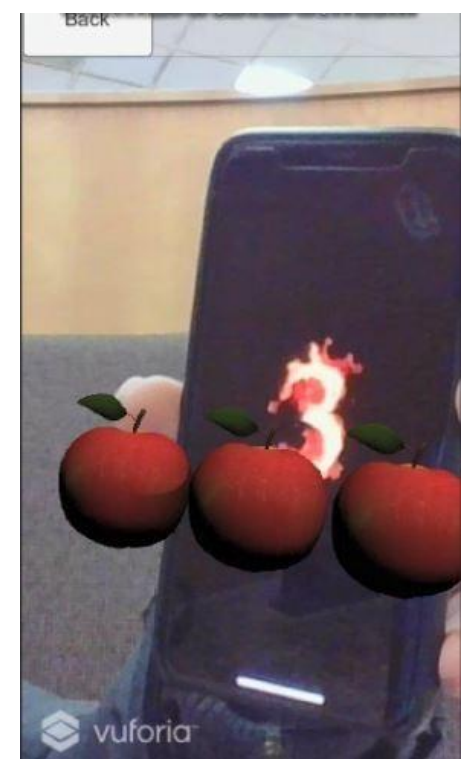

Fig. 6. Vuforia execution of Learning Number.

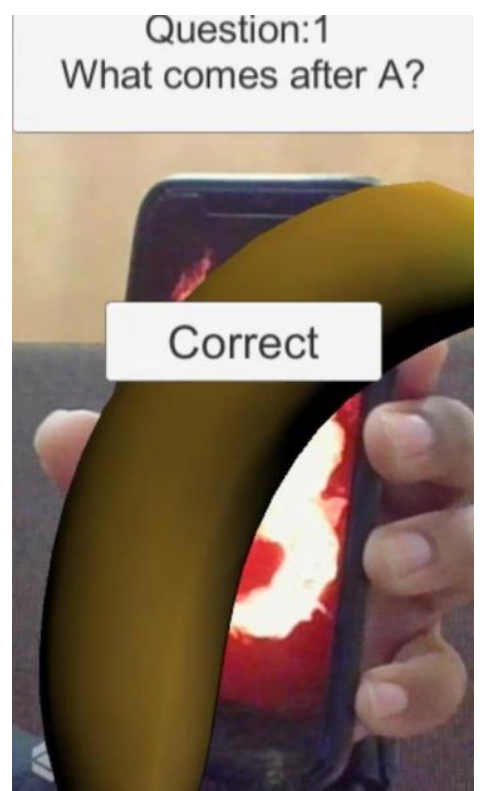

Fig. 7. Question for alphabet module in Examination

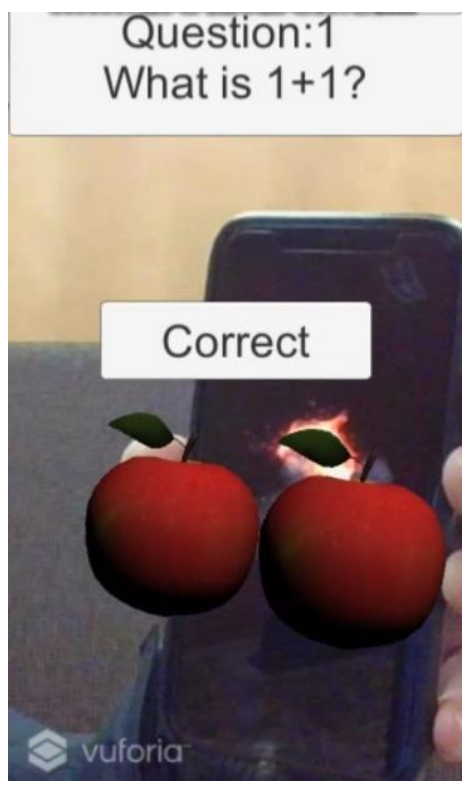

Fig. 8. Question for number module in Examination.

\section{CONCLUSION}

Augmented reality is considered an emerging technology that has been around for years has been making tremendous and astounding growth every year. AR is going to change the shape of research and the world's technology thoroughly shortly in almost every domain. The research we are proposing is primarily based on the education domain that encourages and aiding the children to make learning fun. Also, we are planning to cover both crucial concepts of not only learning but also examination aspects of education. The innovative part is the application of our implementation which is in the form of a game. Moreover, we are making sure to develop a game that is supported by every platform, so we use a dynamic combination of Unity 3D and Vuforia. The augmented reality technology is a bit crude yet and still in its infancy. To conclude, Augmented Reality is revolutionary, and it can take the educational system to a whole new level. Our proposed research in AR is an approach to this modern era by surpassing the limitations of conventional systems.

\section{ACKNOWLEDGMENT}

We would like to express our special thanks of gratitude to Dr Sabah Mohammed who gave us the golden opportunity to do this paper, which also aided us in doing a lot of Research and we came to know about so many new things and explore the novel concepts. Secondly, we are thankful to our university Lakehead university.

\section{REFERENCES}

[1] Yuen, S., Yaoyuneyong, G., Johnson, E.: Augmented Reality: An Overview and Five Directions for AR in Education. Journal of Educational Technology Development and Exchange 4, 119-140 (2011).

[2] Wu, H.-K., Lee, S., Chang, H.-Y., Liang, J.-C.: Current Status, Opportunities and Challenges of Augmented Reality in Education. Computers and Education 62, 41- 49 (2013).

[3] Squire, K.: Video Games in Education. International Journal of Intelligent Simulations and Gaming 2, 49-62 (2003).

[4] Dünser, A., Walker, L., Horner, H., Bentall, D.: Creating Interactive Physics Education Books with Augmented Reality. In: Proceedings of the 24th Australian Computer-Human Interaction Conference, pp. 107-114 (2012). 
[5] Redondo, E., Fonseca, D., Sánchez, A., Navarro, I.: New Strategies Using Handheld Augmented Reality and Mobile Learning-teaching Methodologies, in Architecture and Building Engineering Degrees.

Procedia Computer Science 25, 52-61 (2013)

[6] Iwata, T., Yamabe, T., Nakajima, T.: Augmented Reality Go: Extending Traditional Game Play with Interactive Self-Learning Support. In: IEEE 17th International Conference on Embedded and Real-Time Computing Systems and Applications, pp. 105-114 (2011).

[7] Chang, K.-E., Chang, C.-T., Hou, H.-T., Sung, Y.-T., Chao, H.-L., Lee, C.- M.: Development and Behavioral Pattern Analysis of a Mobile Guide System with Augmented Reality for Painting Appreciation Instruction in an Art Museum. Computer and Education 71, 185-197 (2014).

[8] Zhang, J., Sung, Y.-T., Hou, H.-T., Chang, K.-E. : The Development and Evaluation of an Augmented Reality-Based Armillary Sphere for Astronomical Observation Instruction. Computer and Education 73, 178-188 (2014).

[9] B. A. Koca, B. Çubukçu and U. Yüzgeç, "Augmented Reality Application for Preschool Children with Unity 3D Platform," 2019 3rd International Symposium on Multidisciplinary Studies and Innovative Technologies (ISMSIT), Ankara, Turkey, 2019.

[10] Jančaříková, Kateřina and Severini, Eva. "Uses of Augmented Reality for Development of Natural Literacy in Pre-Primary Education." In Augmented Reality in Educational Settings, pp.24-55. Brill Sense, 2019.

[11] L. Lee, C. Chau, C. Chau and C. Ng, "Using Augmented Reality to Teach Kindergarten Students English Vocabulary," 2017 International Symposium on Educational Technology (ISET), Hong Kong, 2017, pp. 53-57. 